\title{
Erratum to: Vagueness, tolerance and contextual logic
}

\author{
Haim Gaifman
}

Published online: 7 May 2010

(C) Springer Science+Business Media B.V. 2010

\section{Erratum to: Synthese (2010) 174:5-46 DOI 10.1007/s11229-009-9683-9}

During the production of the article, the author overlooked some errors. Below you will find the corrected text.

Page 5, line 4-5 in the abstract should read:

One is tolerance: the insensitivity of predicates to small changes in the objects of predication (a one-foot increment of a walking distance is a walking distance).

Page 6, line 6-8 should read:

A walking distance is still a walking distance if we increment it by one foot (but not by 5 miles); a child is still a child one hour later (but not 5 years later); and so on.

As of page 7 line 3 the words borderline vagueness should read:

borderline-vagueness

Page 8, line 1, first paragraph of Sect. 2: the line should read:

In ordinary usage, 'vagueness' is a broad term that covers an assortment of loosely connected linguistic phenomena: imprecision, fuzziness, ambiguity, obscurity, lack of specificity (hence the expression 'vague generalities'), and their like.

Page 8, the last line of the first paragraph of Sect. 2 should read:

The indeterminateness, moreover, does not mean that the question is out of order (as would be a category mistake, like asking whether number 3 is happy); the question is appropriate, and unambiguous, but the semantics does not seem to decide it.

Page 16, line 7-4 from the bottom should read:

The online version of the original article can be found under doi:10.1007/s11229-009-9683-9.

H. Gaifman $(\varangle)$

Philosophy Department, Columbia University, New York, NY 10027, USA

e-mail: hg17@columbia.edu 
Here, 'derivation' is defined in a standard way, using a standard system based on modus ponens and universal generalization; a wff is used in a derivation if it is either equal to, or is a component of a wff occurring in the derivation.

Page 17, the last line before Sect. 3.2 should read:

The proof of the Sorites contradiction fails, because it requires an unfeasible context and in unfeasible contexts a tolerant predicate loses its tolerance: it has some sharp cutoff. But unfeasible contexts do not arise in practice, as long as the predicate is not explicitly sharpened.

Page 21, line 14-15 should read:

We can add a formula that expresses the statement that $C$ is feasible, as a conjunct to the antecedent of (TC*); this will give us the scheme: $C$ is feasible $\wedge N P(x, y) \rightarrow$ $[C](P(x) \rightarrow P(y))$.

Page 23, the first line of Sect. 3.3.1 should read:

The non-logical vocabulary includes also context-independent predicates and, possibly, function symbols.

Page 26, footnote 18. The last two lines should read:

CSL, i.e., the sentential fragment of TCL, is much more expressive than CSL\# and its deductive system is an extension of classical logic, which CSL\# is not.

Page 29, line 8-7 above Sect. 3.4.1 should read:

It can be done, using the fact that $(1)$ is a $\prod_{1}^{0}$ sentence.

Page 31, line 3-6 second paragraph of Sect. 3.5 should read:

Note that $\alpha$ may refer to $X$, even when $f(X)$ has no effect on $\alpha$ 's truth-value; for example, the truth-value of a logical truth containing $P$ is independent of the interpretation, but the sentence refers to some contexts; $\forall u(P(u) \rightarrow(u))$ refers to all one-element contexts.

Page 32, line 1-3 should read:

Then a sentence $\alpha$ refers to the context $X$, iff $X$ is the set of values (in $|\mathcal{M}|$ ) of some context, $C \in \operatorname{cont}(\alpha)$, under some assignment of values to the variables occurring in $C$.

Page 34, line 5-7 of the second paragraph should read:

Moreover, one's hesitation may be due to semantic indeterminacy, therefore an expression of confidence can mean that this case is not one of semantic indeterminacy, i.e., is not a borderline case.

Page 35, line 6 of the third paragraph should read:

This corresponds to one reading of the inconsistent notation in Evans (1978). My notation, which differs from that of various authors, is motivated by the need for a convenient, natural dual for $\Delta$.

Page 37, line 7-5 should read:

If every world is accessible from every world, then, in each of these worlds, $\Delta P$, $\mathrm{B} P$, and $\neg P$ have, respectively, the extensions $\{0,1\},\{2,3\}$, and $\{4, \ldots, 8\}$, and each is sharp (i.e., has an empty borderline).

Page 44, last two lines of the third paragraph should read:

The only discovery is the technique by which models that deliver certain effects can be constructed. 